\title{
Muséologies
}

Les cahiers d'études supérieures

\section{Le petit musée des migrations de Lampedusa}

\section{Giovanna Costanza Meli}

Volume 8, numéro 2, 2016

Les nouveaux paradigmes

URI : https://id.erudit.org/iderudit/1050759ar

DOI : https://doi.org/10.7202/1050759ar

Aller au sommaire du numéro

Éditeur(s)

Association Québécoise de Promotion des Recherches Étudiantes en

Muséologie (AQPREM)

ISSN

1718-5181 (imprimé)

1929-7815 (numérique)

Découvrir la revue

Citer ce compte rendu

Costanza Meli, G. (2016). Compte rendu de [Le petit musée des migrations de Lampedusa]. Muséologies, 8(2), 47-61. https://doi.org/10.7202/1050759ar

Tous droits réservés (C Association Québécoise de Promotion des Recherches Étudiantes en Muséologie (AQPREM), 2018
Ce document est protégé par la loi sur le droit d'auteur. L’utilisation des services d'Érudit (y compris la reproduction) est assujettie à sa politique d'utilisation que vous pouvez consulter en ligne.

https://apropos.erudit.org/fr/usagers/politique-dutilisation/ 
Carnet un

\section{Le petit musée des migrations de Lampedusa}

Giovanna Costanza Meli 
Giovanna Costanza Meli est candidate au doctorat en histoire de l'art à l'université La Sapienza à Rome et commissaire d'exposition. Elle a fondé en 2007 Isole, une association à but non lucratif promouvant les pratiques d'art public participatives et communautaires, ainsi que le développement à l'échelle globale, d'une meilleure compréhension d'imaginaires nouveaux. L'association a réalisé plusieurs projets de recherche dans le champ muséal qui se sont matérialisés, dont un programme de résidence d'artistes internationaux et la création du musée des Migrations de Lampedusa. 
On m'a plusieurs fois demandé de raconter et de contextualiser de façon critique le projet d'archivage entrepris par le petit musée des migrations de Lampedusa. Au-delà de la rhétorique et des lieux communs sur l'accueil et l'immigration d'une part, et sur la dimension sociale des institutions muséales d'autre part, je crois qu'il s'agirait en réalité d'une entreprise complexe, méritant plus de place et beaucoup d'écrits. Je me limiterai donc ici à avancer quelques points de réflexion qui me sont venus en discutant avec les acteurs de cette expérience, dont la parole est la mieux à même de transmettre le sens et les émotions qui la traversent. Commençons par le premier terme complexe: le musée.

Si l'on considère le musée comme une institution, il semble naturel d'imaginer des corrélations sémantiques avec une tradition, une collection, un comité scientifique, une structure administrative, une représentation culturelle plus ou moins codifiée, une politique culturelle plus ou moins connotée qui interrogerait le contexte et rechercherait un public auquel montrer tout cela. Si l'on se réfère au débat interne qui agite les institutions muséales contemporaines, on pense aux démarches plus innovantes d'intégration et de médiation culturelle, aux réflexions sur le rôle public et social du musée et aux recherches entreprises par les services pédagogiques des musées. Autant d'éléments qui ont évidemment leur place dans l'imaginaire des historiens et des chercheurs, de ceux qui réfléchissent aux dynamiques d'hybridation et de métissage que le musée doit aujourd'hui traiter ou au renversement que ces concepts induisent par rapport aux représentations de l'identité et de l'altérité.

Mais qu'en est-il de Lampedusa? Qu'est-ce que cette île et sa population ont à voir avec les débats scientifiques et culturels sur le musée? Qui sont les protagonistes de cette histoire? Qui a mis cette collection sur pied et quels objets regroupait-elle? Que racontent les pièces et les traces recueillies? Quelle identité et quelle altérité? Nous parlons de mémoire mais aussi des significations étranges et inattendues qui se cachent derrière ce mot. Nous faisons le récit d'un parcours, celui de la construction d'une mémoire à la fois collective et individuelle, en dehors de toute narration conventionnelle et loin des structures discursives dominantes.

Examinons d'abord qui sont les protagonistes de notre «musée ». Askavusa, devenu aujourd'hui un collectif de militants, était au tout début de cette histoire une association culturelle, mais aussi un groupe d'amis. À Lampedusa, Giacomo Sferlazzo, chanteur-compositeur, a commencé à ramasser des objets, des morceaux de bois, des épaves de bateaux naufragés ou démantelés; autant de matériaux retrouvés dans ce qui a été surnommé «le cimetière des bateaux ». Lui et ses amis ont sauvé de la destruction ces objets abandonnés dans des décharges. Askavusa est aussi l'un des acteurs qui s'est le plus engagé ces dernières années pour apporter aide et assistance aux milliers de jeunes, de femmes, d'enfants, d'hommes qui ont survécu à leur voyage à travers la Méditerranée, à bord de ces bateaux-épaves qui, 
en l'absence de couloirs humanitaires et d'une organisation internationale, constituent leur seul moyen de fuir la guerre.

J'ai écouté et observé les protagonistes de cette histoire. J'ai longuement discuté avec eux pour comprendre comment l'on affronte de telles situations d'urgence et comment l'on réagit devant le débarquement régulier de migrants qui traversent la mer Méditerranée et qui arrivent à Lampedusa, un coin d'Europe à leurs yeux, un nouveau départ. Au cours de ces discussions, j'ai découvert un univers de solidarité et d'engagement citoyen mais aussi une grande lucidité sur les causes et les responsabilités à l'origine des actuelles migrations de masse, une conscience aiguë de leur propre rôle, de l'importance de l'action collective et du témoignage. Dans une interview réalisée en 2012, j'ai demandé à Giacomo ce qui l'avait poussé à recueillir et à conserver ces objets (morceaux d'épaves, corans, bibles, lettres adressées aux proches, amulettes de voyage, vêtements, effets personnels) qui représentent la seule trace laissée par ces milliers d'individus et de vécus différents, que personne ne connaît, et que les médias nous présentent comme une masse indifférenciée. La recherche de la mémoire, ai-je compris alors, est un cheminement individuel qui commence par soi, par ses propres racines, et qui se transforme dans la rencontre avec l'autre.

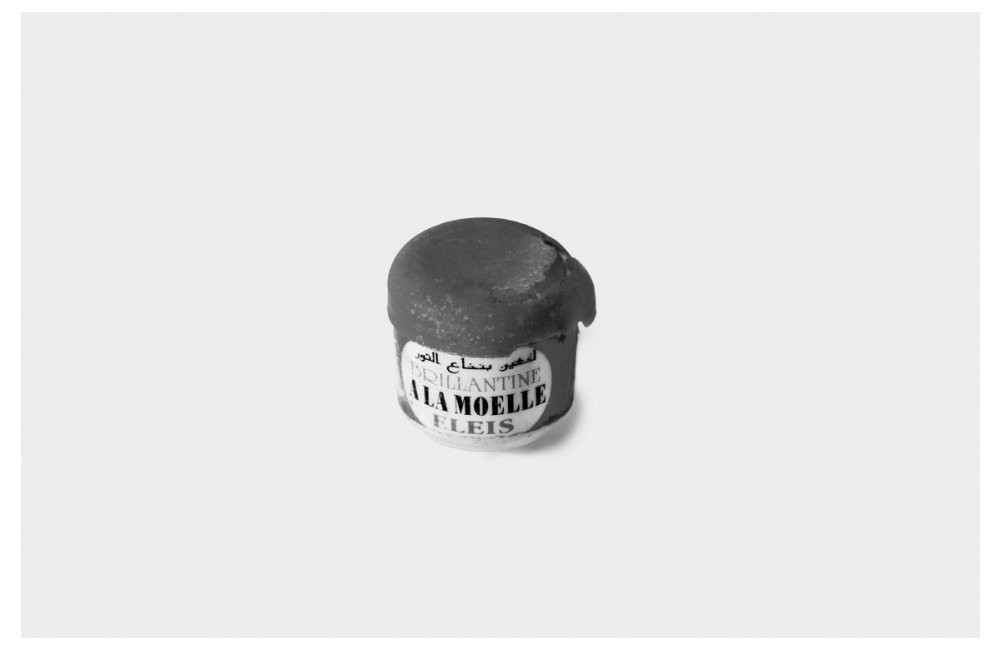

Figure 2

Brillantine pour les cheveux, de l'archive du projet du Musée des migrations, Lampedusa. () Mario Badagliacca.

[Photographe: Mario Badagliacca]

" La première fois que je suis allé au cimetière des bateaux, je cherchais de quoi être étonné au milieu des ordures, comme il m'arrivait souvent de le faire. J'ai toujours nourri une certaine curiosité envers les objets. Je me souviens des salles à manger de mes grands-parents avec les buffets remplis d'objets ayant tous une histoire, tous chargés de souvenirs Mais lorsque pour la première fois, au milieu de morceaux 
de bois défoncés, j'ai trouvé un paquet de lettres, de photos, de textes sacrés, ce que j'ai ressenti n'avait rien à voir avec ce que j'avais ressenti jusqu'ici au cours de mes pérégrinations au milieu des choses jetées. C'était comme si j'avais trouvé ce que j'avais longtemps cherché, j'avais trouvé le témoignage d'une humanité enveloppée dans le mystère de la vie et peut-être le mystère lui-même. C'était comme prendre part à I'histoire de l'humanité tout entière, comme avoir découvert les pyramides d'Égypte, comme s'engager sur une route menant, tout au bout, à une promesse de lumière et de libération mais dont le parcours est jonché de douleur et d'injustice'. "

Une nouvelle approche de la mémoire et de l'identité se forme alors dans l'esprit de Giacomo et pour l'association Askavusa qui l'accompagne depuis quelques années dans cette recherche et cette mission. Ce qui m'a tout de suite frappée, c'est que ceux qui percevaient avec force ce qui fonde le processus même de muséalisation étaient des gens ordinaires, et pas un organisme ou une institution. Je me demandais: pourquoi les jeunes et la population d'une île si tourmentée et si souvent oubliée ont-ils ressenti la nécessité de " muséaliser »ce qu'ils avaient trouvé? Quel est ce besoin inhérent au récit, au témoignage et à la recherche d'identité qui croît vis-à-vis de la mémoire d'autres peuples, d'autres personnes, de ceux qui voyagent et qui passent? Que peut représenter cette mémoire qui se réalise dans la rencontre entre histoires et récits divers?

Voici ce que Giacomo m'a répondu.

«Le musée des migrations peut être une première approche pour inscrire le développement de l'île de Lampedusa sur une voie choisie, qui ne soit pas le fruit du hasard, une voie qui pourrait être un exemple pour le monde entier, où l'on considère l'intégration comme une ressource non seulement juste mais aussi inévitable D'ici une trentaine d'années, les enfants, petits-enfants, frères et sœurs de ceux qui sont aujourd'hui vus comme des clandestins seront des citoyens italiens et européens à part entière et nous avons le devoir de conserver les témoignages de ces mouvements migratoires, sinon ils risquent de finir aux oubliettes. Nous avons le devoir de sauver au moins les bateaux, les corans et les bibles, les vêtements, les chaussures, les documents et tous les petits objets que la mer nous a redonnés et qui racontent, et raconteront aux générations suivantes, ces tragédies, ces espoirs, ces rêves qui, bien souvent, se noient dans les profondeurs de la Méditerranée ${ }^{2}$.

C'est ce qui m'a convaincue, sans conditions ni objections, à accepter la demande d'Askavusa de participer à la réalisation d'un projet qui 
permettrait de faire progresser cette collection déjà intéressante, afin de comprendre, défendre et conserver ces souvenirs, dans le respect de ceux auxquels ils ont appartenus. C'est de là qu'est partie la volonté d'une nouvelle façon de faire, afin d'approfondir la réflexion autour du thème du musée, sans atermoiements, et dans l'optique de construire quelque chose d'expérimental et de commun, qui intégrerait avant tout les migrants dans la lecture de l'histoire en cours.

L'association Askavusa qui, au fil des ans, a échafaudé plusieurs hypothèses d'aménagement, de divulgation, de réélaboration artistique et d'étude des objets trouvés en s'entourant d'artistes, d'associations culturelles et de militants politiques, a émis le souhait de réaliser un projet plus structuré et conçu à long terme. Se faisaient de plus en plus sentir, en effet, la nécessité et l'urgence d'une intervention de récupération pour sauvegarder cette collection, en faisant appel à des compétences spécifiques. Le mauvais état de conservation de la plupart des matériaux documentaires, en particulier des documents papier et des photos, ne permettait pas de faire une consultation détaillée, nécessaire à l'identification de la nature de chaque document.

\section{Figure 3}

Coran, de l'archive du projet du Musée des migrations, Lampedusa. @ Mario Badagliacca.

[Photographe: Mario Badagliacca]

Pour ce faire, nous avons mis au point une méthode de travail interdisciplinaire et horizontale, réunissant artistes et experts parmi lesquels des anthropologues, des géographes, des éditeurs, afin de multiplier le plus possible les angles d'attaque. 


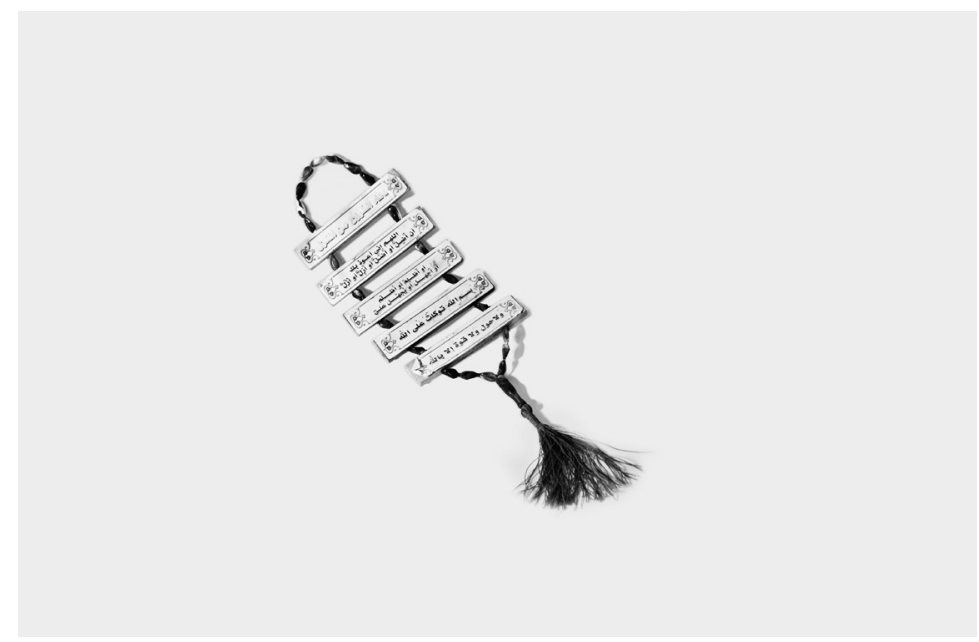

Figure 4

Chapelet populaire avec du texte superposé, de l'archive du projet du Musée des migrations, Lampedusa. (c) Mario Badagliacca.

[Photographe: Mario Badagliacca]

\section{Le projet du musée et ses protagonistes}

L'association Isole, que je dirige aux côtés de Barbara D'Ambrosio depuis 2007, est une unité de recherche sur la relation entre l'art contemporain et le territoire, au sein des différentes formes d'art en espace public, d'art relationnel, participatif et in situ. L'envie réciproque de collaboration entre nous et le collectif Askavusa pour créer le Musée de Lampedusa tient principalement à notre réflexion approfondie depuis plusieurs années de la signification même du territoire. L'expérience du territoire se situe à différents niveaux - celui de la connaissance rationnelle, celui de l'empathie, qui sont parfois opposés l'un à l'autre, et celui de la poésie (qui intervient dans l'élaboration artistique) - qui doivent être libres d'interagir ou de se développer parallèlement. Tout le travail d'Isole se fonde sur l'idée que la valeur de l'intervention artistique, qui est notre principal objet de travail, réside dans la capacité à interagir avec l'imaginaire des lieux et avec les composantes sociales, et pas toujours rationnelles, de la perception territoriale. Dans tous nos projets réalisés jusqu'à présent, les artistes ont été invités à entreprendre un voyage, participant ainsi à une lecture complexe de la réalité dans laquelle nous travaillions, en termes de collectif et d'atelier. De même à Lampedusa, notre intervention aurait été tournée aussi bien vers la dimension concrète et scientifique que la création d'archives et d'une collection implique aujourd'hui, que vers l'organisation d'une série de résidences d'artistes internationaux venus interagir poétiquement et esthétiquement dans le projet.

À partir de 2011, la collaboration de l'association Isole s'est renforcée, avec l'aide d'une autre organisation importante qui avait déjà participé aux initiatives sur le territoire: I'association AMM, Archivio delle Memorie Migranti de Rome. 
S'est alors constitué le groupe de travail qui allait donner l'orientation du musée, principalement sous l'impulsion d'un homme exceptionnel qui a mené ce projet avec une immense passion et un grand professionnalisme. Le professeur Giuseppe Basile, restaurateur de renommée internationale fort d'une longue expérience de la muséologie contemporaine, avait déjà entendu parler de l'initiative des jeunes de Lampedusa et, emballé par cette idée, il avait décidé de s'y consacrer, conscient que cette collection d'objets était une grande ressource pour l'humanité. Basile, après s'être occupé des chefs-d'œuvre de l'art moderne italien, après avoir été un pionnier de la restauration d'objets d'art contemporain et l'une des références mondiales en la matière, a décelé dans cette collection une valeur humaniste essentielle et c'est pour cette raison qu'il a choisi de s'y consacrer dans les dernières années de sa vie. Sous son influence, le projet du musée a pris un tour nouveau, avec une dimension expérimentale, innovante vis-à-vis des habitudes propres aux musées démo-ethno-anthropologiques, et l'intention de se distinguer des aménagements commémoratifs plus conventionnels des " musées de la mémoire ». Nous percevions d'un côté l'importance de transmettre à la collectivité un patrimoine culturel et historique mais aussi, de l'autre, la nécessité de soustraire les objets et leur image au risque de fétichisation, de réduction à un simulacre grotesque d'un événement déshumanisé, la mort de femmes, d'hommes et d'enfants dévoilée et étalée sans pudeur. De ces considérations est né notre choix de définir un parcours de recherche et une collection raisonnés, qui ajouteraient la fonction de sauvegarde à la traduction et l'étude des documents catalogués, à faire avec les migrants eux-mêmes, afin de restituer leur histoire et les parcours de vie des personnes.

"Le projet du musée faisait face à une série de questions dont il fallait tenir compte dans le délicat travail d'élaboration et d'exposition. Il y avait le risque que ces objets, une fois séparés du processus qui les avait portés jusqu'ici dans cet état, servent uniquement à alimenter des formes de voyeurisme, et que le fait de ne présenter que le résultat du processus impose des modalités vétustes de muséalisation, considérées comme vivisection objectivante. L'une des problématiques principales était le rapport particulier de présence/absence entre objets et migrants, à la fois point de friction et agent de fermentation de la dimension politique de l'initiative. Tandis que très souvent, les musées « ethnographiques » naissent de la nécessité de répondre à la disparition imminente d'un phénomène ou de la volonté de réactualiser quelque chose qui n'est plus " vivant », le projet de musée de Lampedusa se trouvait devant la situation inverse. Parfois en effet, les objets sont ramassés à moins d'une centaine de mètres d'autres migrants pris en charge par les autorités, une proximité spatiale qui ne s'accompagne pas toujours de la possibilité de rencontres humaines ${ }^{3} »$.

3 GATTA Gianluca, Lo sguardo antropologico (Le regard anthropologique), (MOSCA MONDATORI Arnoldo Mondadori, CACCIATORE Alfonso, TRIULZI Alessandro dir.), La Bibbia e il Corano di Lampedusa (Bible et Coran à Lampedusa), Milan, Éditrice La Scuola, 2013, p. 148. 


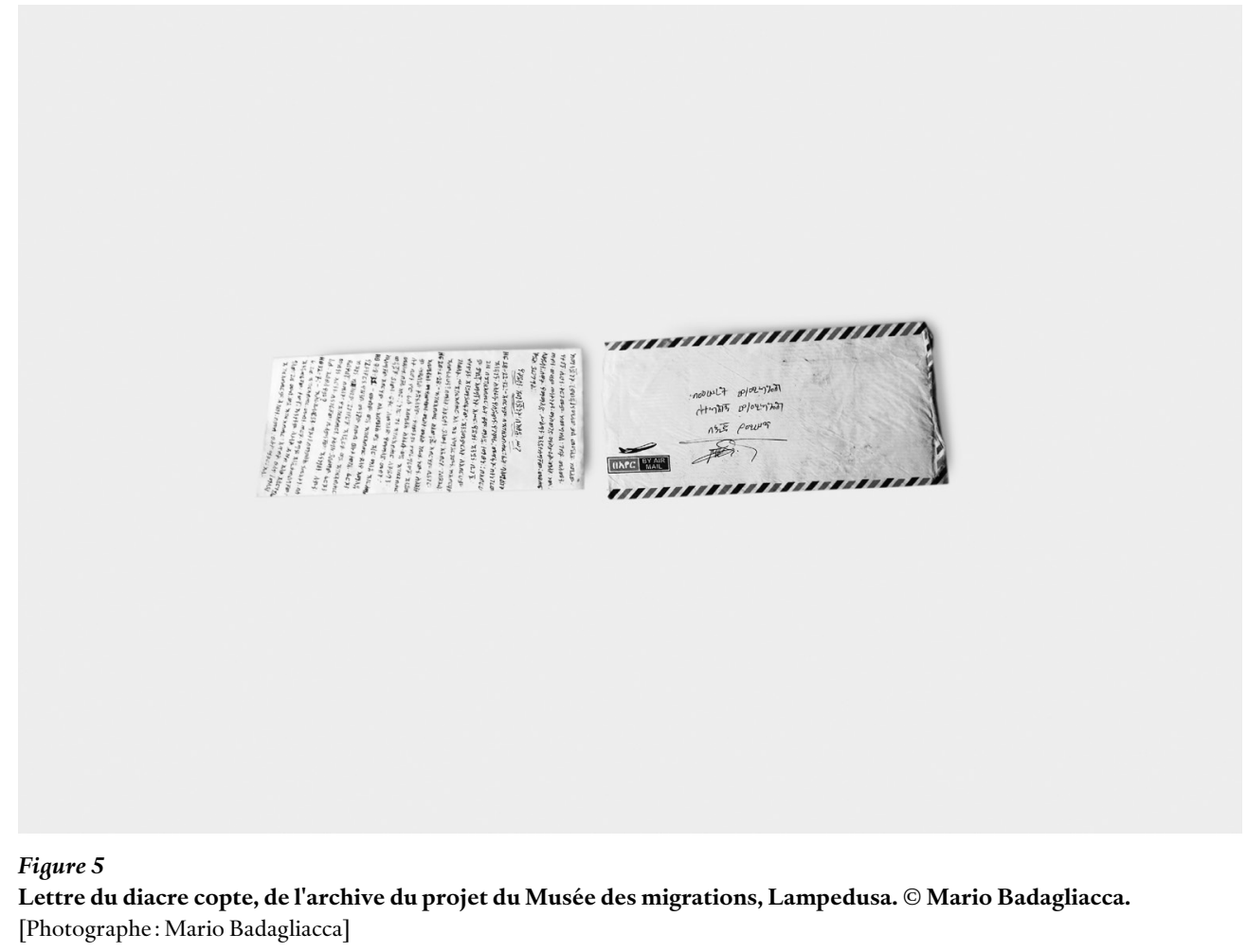

\section{L'intervention sur la collection}

L'association Isole s'est occupée, avec le professeur Basile, de la construction du catalogue, de la restauration et de l'exposition du premier groupe de pièces sélectionnées. Le projet d'archivage a été pensé par Giuseppe Basile comme une étape essentielle d'un processus qui devait au préalable passer par une phase de restauration, de sauvetage des matériaux papier, puis de numérisation en vue de leur étude. Après quoi seulement, les objets auraient été montrés dans un lieu dédié, en respectant les conditions de sauvegarde. Tout ce processus, que je décrirai dans ses grandes lignes, n'était pas le but final, mais chacune des étapes de ce travail était considérée comme préliminaire et indispensable aux objectifs que nous nous étions fixés. Les pièces ont été classées de façon à ce qu'elles puissent témoigner d'une tragédie réellement vécue par des personnes obligées de fuir leur pays, et à ce qu'elles soient aussi un document politique du résultat des choix historiques, économiques et culturels à l'origine des mouvements migratoires en Méditerranée. L'objectif était en réalité d'identifier les propriétaires pour leur restituer, à eux ou à leur famille, dans un futur proche, non seulement l'histoire mais aussi les objets eux-mêmes, les albums photos de mariage, les lettres personnelles. 
Le système que nous avons mis en place s'est donc articulé en différentes étapes. La première consistait à faire l'inventaire des objets in situ, en vue de leur conservation. Mais avec quels instruments? Selon quels critères? Par où commencer et quelle priorité donner à nos choix? Avec Basile, nous avons effectué une première sélection qui constitue le noyau initial de la " collection »: quelques dizaines d'objets choisis avant tout en raison de leur capacité à représenter la catégorie à laquelle ils appartenaient, pour ainsi synthétiser la complexité et la diversité des matériaux récupérés. Puis, en fonction de l'état des objets, nous mettions de côté ceux dont la détérioration nécessitait une " lecture " plus approfondie ou ceux destinés à une première série d'interventions de conservation et de restauration. En fonction de ces critères, il a été possible d'obtenir deux séries: l'une constituée d'objets directement documentaires, c'est-à-dire de matériaux papier diversement interprétables (lettres, cartes postales, cahiers, photos, notes, papiers d'identité, etc.), et l'autre constituée d'objets indirectement documentaires, choisis par catégories représentatives (outils, vêtements, récipients, nourriture, etc.).

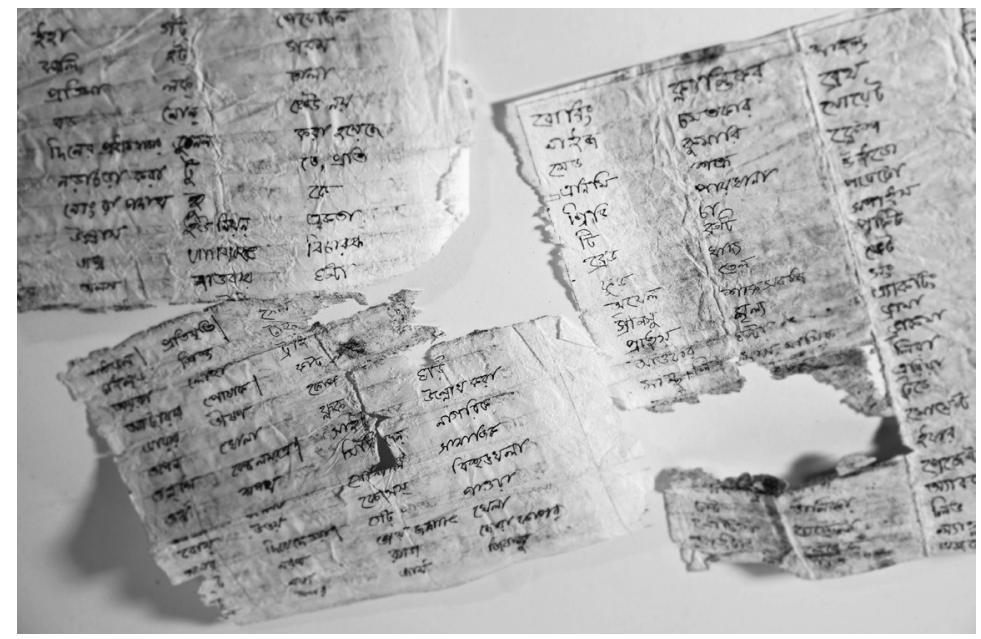

\section{Figure 6}

Amulette magique avec une écriture " à lunettes ", les phases de restauration, de l'archive du projet du Musée des migrations, Lampedusa. () Giovanna Costanza Meli. [Photographe: Giovanna Costanza Meli].

Nous avons procédé au catalogage à proprement parler en imaginant une fiche de catalogue ad hoc comme un instrument pouvant contenir et classer des informations analytiques, techniques et conservatives, mais aussi des éléments de contexte, même pour des objets aussi variés. De façon expérimentale, la fiche a été élaborée en croisant les intitulés des fiches $O A$ et $D$ d'une part, utilisées par le Ministère italien respectivement pour œuvres d'art et dessins, et celui de la fiche concernant les biens démo-ethno-anthropologiques d'autre part. A émergé, dès le début des travaux, la nécessité de réunir de nombreuses compétences pour la lecture et l'interprétation des objets 
que nous cataloguions, à travers un travail d'équipe réunissant les migrants eux-mêmes, surtout pour la reconnaissance, la compréhension et la traduction des outils et des matériaux papier, Basile et l'association Isole, des chercheurs des Archives des mémoires migrantes (Archivio delle Memorie Migranti) pour l'identification et la traduction des matériaux et l'association Askavusa qui les avait récupérés, témoin direct des arrivées de migrants, capable de restituer le contexte et les conditions de récupération des objets, même au sein de dynamiques sociales.

Pour le travail de traduction, le groupe opérationnel a pu bénéficier de diverses aides scientifiques, principalement de la part de chercheurs des universités de Naples L'Orientale, Rome La Sapienza et Florence, et d'experts du Centre Al-Quds de Palerme qui, dès le début, ont voulu apporter leur contribution au projet de Musée des migrations de Lampedusa.

Cette pluralité des contributions ainsi que la participation des migrants à différents stades du projet a constitué pour tout le groupe aussi bien le présupposé méthodologique d'une façon de faire partagée et horizontale qu'un élément indispensable à la réussite même du projet.

Comme l'a souligné à plusieurs reprises l'anthropologue Gianluca Gatta qui a fait partie du comité scientifique dès le départ, nombreux étaient les dangers inhérents à un projet prenant place dans un territoire tellement actuel et traversé de fortes contradictions. II était important de savoir reconnaître ces dangers si l'on voulait pouvoir répondre aux questions qui affluaient constamment au sujet de l'usage et de la valeur des objets trouvés, par rapport aux vies et aux vicissitudes des personnes qui arrivent et qui partent de Lampedusa.

«Pour éviter ces risques, il faut que les personnes porteuses de ces expériences participent activement au processus d'élaboration d'une mémoire migrante à travers les objets, en enrichissant les traces retrouvées d'histoires, de points de vue, d'observations subjectives, d'interprétations. Dans ce sens, le contact avec la matérialité immédiate de ces objets et leur valeur symbolique avait pour but de stimuler une attention différente à l'expérience migratoire, en enrichissant la qualité de l'écoute ${ }^{4}$ ».

Le parcours lancé sous la direction de Basile s'est achevé par les phases de restauration et d'exposition. La restauration a été possible grâce à la précieuse collaboration de l'atelier de restauration de la Bibliothèque centrale de Sicile (Biblioteca Centrale della Regione Siciliana), qui a soutenu le projet en mettant à disposition ses compétences et ses outils pour les premiers matériaux fichés. Il s'agissait de traitements 
de conservation évidemment mais, comme l'a précisé son directeur Francesco Vergara Caffarelli, dans sa lettre de remise des pièces adressée au maire de Lampedusa:

"Les restaurateurs de l'atelier, en accord avec les organisateurs de ce musée naissant, ont considéré que les interventions qu'ils devaient faire n'avaient pas pour but de récupérer l'intégralité des ouvrages, mais seulement de les sauvegarder et d'en assurer la conservation, le but étant de maintenir les pièces dans leur état actuel, reflétant la mémoire qu'elles transmettent ${ }^{5} »$.

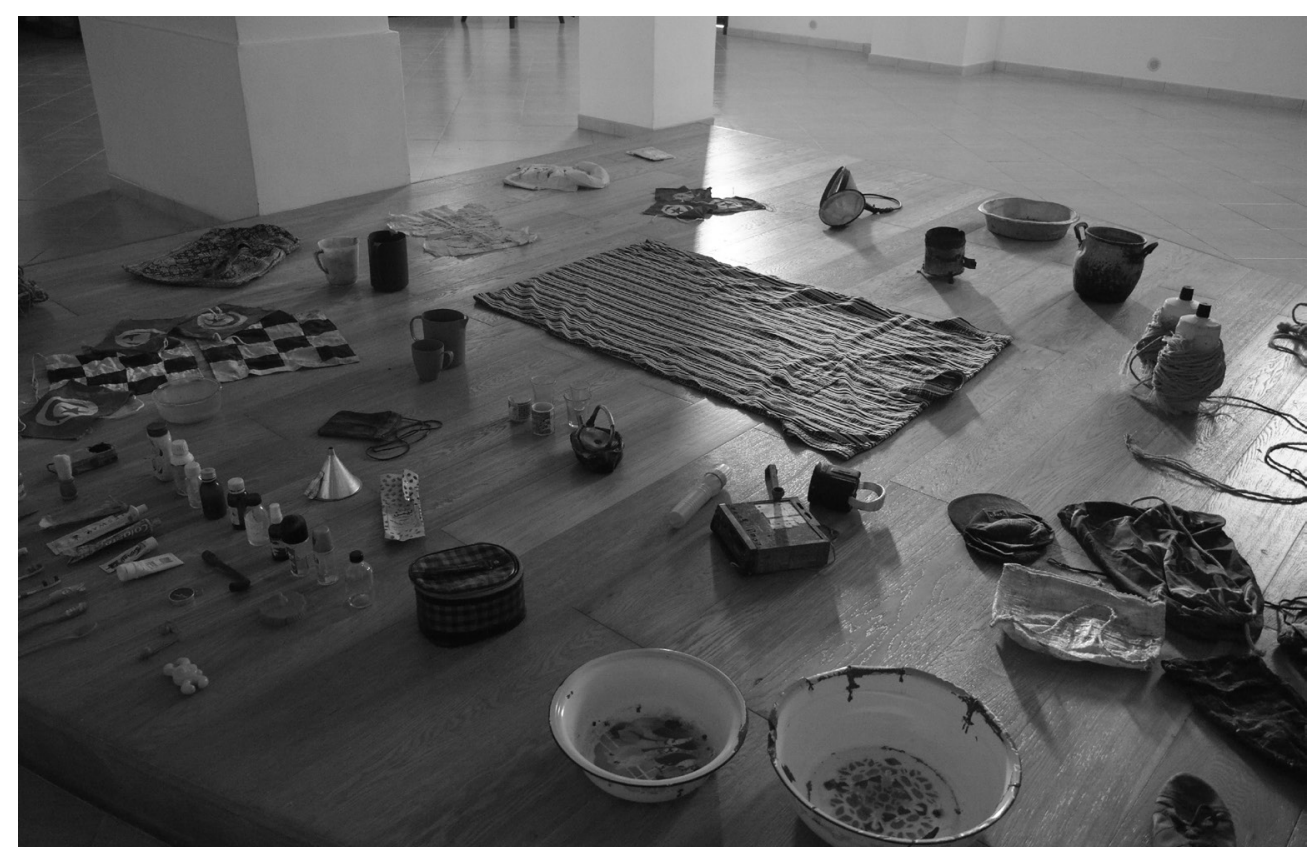

Figure 7

Exposition "Con gli oggetti dei migranti" (Avec les objets des migrants), de l'archive du projet du Musée des migrations, Lampedusa. (c) Giovanna Costanza Meli.

[Photographe: Giovanna Costanza Meli]

\section{L'exposition}

Le 16 juillet 2013, à l'occasion de « Lampedusa in festival », a eu lieu à Lampedusa l'inauguration de l'exposition Con gli oggetti dei migranti (Avec les objets des migrants), sous la direction de l'association Isole. L'exposition était donc la conclusion de cette première phase opérationnelle du projet plus ample de musée/centre de documentation. 
Ce n'était donc pas uniquement une exposition qui présentait la collection d'objets ayant appartenu à des migrants et retrouvés sur l'île, mais quelque chose de plus important sur le plan culturel, scientifique et social. C'est également à cette occasion en effet que l'association Isole a organisé la première résidence d'artiste du programme en conviant sur l'île Emily Jacir. L'artiste palestinienne a été invitée à élaborer un projet qui, à partir des objets exposés, puisse s'étendre à la réalité territoriale de Lampedusa, à son vécu et aux problématiques qui s'entremêlent au malaise de la population, obligée d'affronter des situations d'urgence qui dépassent ses propres possibilités et dans un état d'isolement total par rapport au reste du pays. La résidence de l'artiste n'a pas été programmée à partir d'une idée définie d'intervention; Emily Jacir a eu l'occasion d'appréhender les lieux et les différents événements qui survenaient même fortuitement, sans aucun conditionnement lié à la production de l'œuvre ou à l'utilisation de moyens d'expression particuliers. L'artiste a été accompagnée dans une série de rencontres grâce auxquelles elle a pu découvrir le territoire à travers le récit des habitants et de ceux jouant un rôle particulier dans I'accueil des migrants, notamment dans les moments d'urgence attribuables au surpeuplement, ou le récit des organisateurs d'initiatives culturelles privées comme les archives historiques de Lampedusa. Cette expérience a confirmé à notre intuition que l'art contemporain, qui part d'une écoute des lieux et des histoires pour promouvoir et valoriser le dialogue, peut atteindre une valeur humaniste fondamentale transmise à travers des langages inhabituels qui font vibrer des cordes à la fois personnelles, intimes et collectives.

Au moment où j'écris, l'association Isole ne fait plus partie du comité scientifique du projet de Musée des migrations, car cette équipe n'existe plus aujourd'hui. Une fois passée la première étape du projet, c'est-à-dire l'exposition à Lampedusa du premier groupe d'archives et la première résidence d'artiste, les activités autour de ce projet se sont soudainement arrêtées à l'automne 2013 et le projet a changé de direction.

L'association Askavusa, qui ne se reconnaissait plus dans les formes institutionnelles, s'est dissoute, avant de se transformer en collectif de militants qui mène sur place une lutte politique et idéologique contre l'instrumentalisation médiatique des migrations, avec pour objectif de mettre à jour les dynamiques géopolitiques qui sous-tendent un phénomène d'envergure mondiale. Le choix du collectif s'est étendu au projet de musée avec un virage radical par rapport à la vision jusque-là partagée. Les principes relatifs à la conservation, la traduction et la sauvegarde des objets et textes recueillis ont été remis en question au point d'amener le collectif à créer un nouveau projet appelé "Porto M », aux objectifs presque opposés. Dans le manifeste de cette nouvelle entité créée sur l'île début 2014 est exprimée l'intention de soustraire la collection sauvée de la décharge à de possibles lectures coloniales et aux processus d'archivage et de muséalisation qui figent les objets dans le temps en les scellant. Des peurs et des réserves qui, bien qu'elles aient constitué le point de départ à la création d'un 


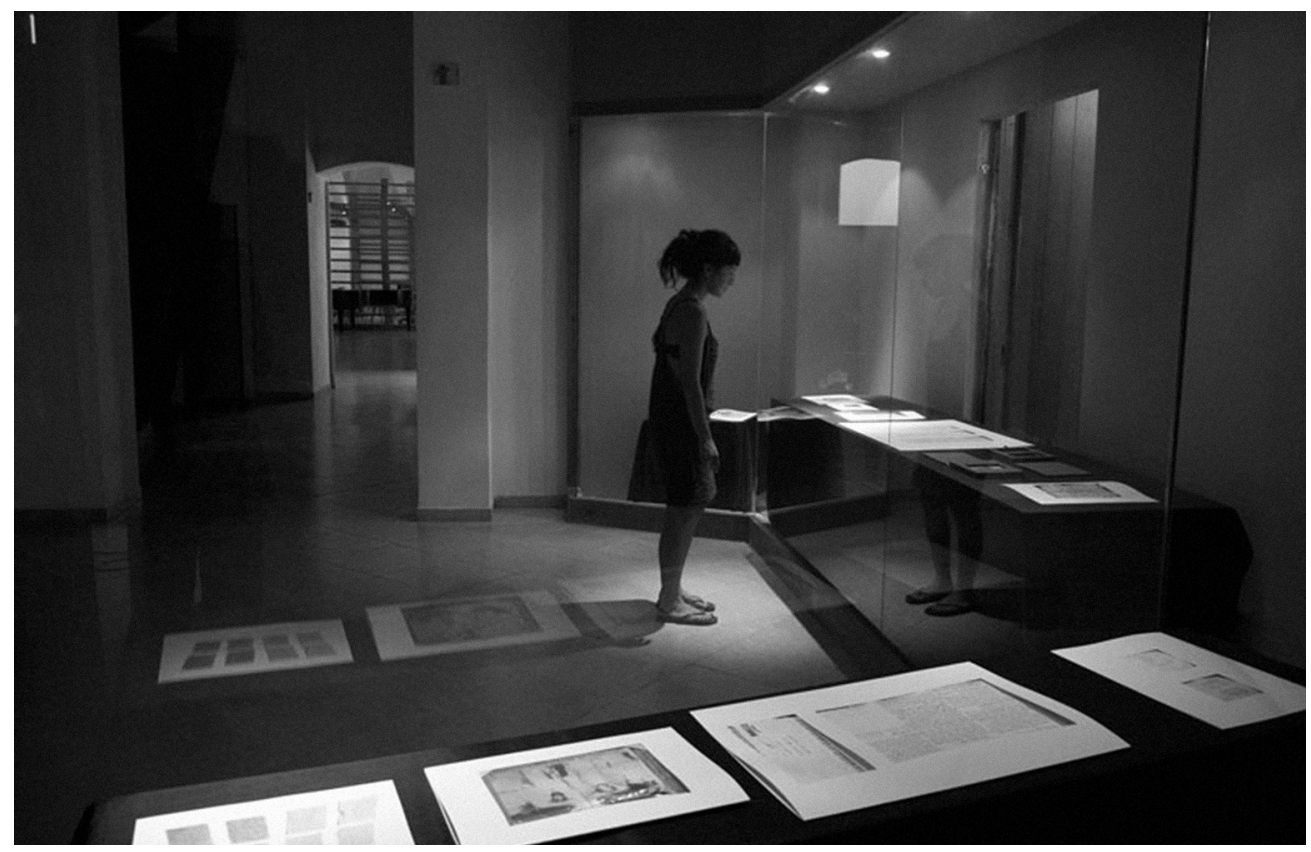

Figure 8

Exposition "Con gli oggetti dei migranti" (Avec les objets des migrants), de l'archive du projet du Musée des migrations, Lampedusa. () Giovanna Costanza Meli.

[Photographe: Giovanna Costanza Meli].

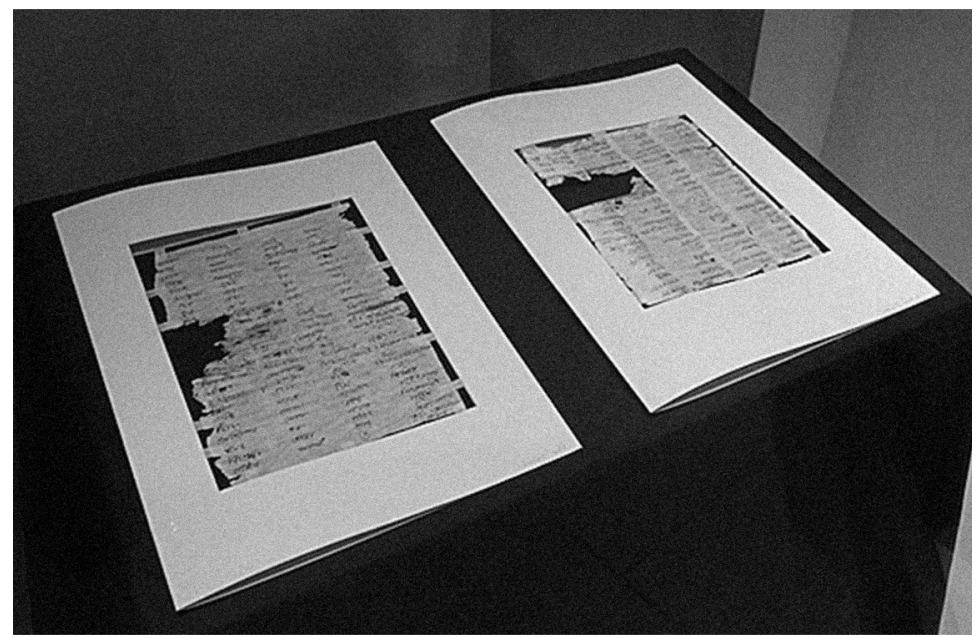

Figure 9

Glossaire bangla-anglais, de l'exposition "Con gli oggetti dei migranti" (Avec les objets des migrants), de l'archive du projet du Musée des migrations, Lampedusa. (c) Giovanna Costanza Meli.

[Photographe: Giovanna Costanza Meli] 
centre de documentation comme voix critique et sévère par rapport aux politiques migratoires, continuaient de toute évidence à être un sujet d'incompréhension et de critique à l'intérieur du projet. Une critique que je trouve intéressante à prendre en compte, aujourd'hui plus que jamais, par rapport à mon questionnement de départ, à savoir la valeur et le destin de nouvelles approches et de nouvelles pratiques concernant le musée. Et voici quel est l'un des destins possibles d'une approche expérimentale, avec la rencontre de visions diverses, sur un thème et dans un contexte d'une grande complexité: l'échec (au moins dans un premier temps).

Les objets sur lesquels le groupe de travail s'est penché dans la phase de construction du " musée " sont restés enfermés dans des caisses conservées à la mairie de Lampedusa, par le maire Nicolini qui a choisi de ne jouer aucun rôle dans cette impasse. Ce qui aurait pu, et selon moi dû, être reconnu au plus vite comme une collection d'objets inscrite au patrimoine de l'humanité est ainsi restée muette, enfermée dans des boîtes inappropriées, et destinée, dans l'incurie dans laquelle elle se trouve, à être oubliée et refoulée de la mémoire collective. Et c'est peut-être ce qu'il y avait de plus efficace à faire pour favoriser l'uniformisation des représentations culturelles que l'on voulait justement combattre avec force.

À l'inverse, la collection conservée par Askavusa auprès de Porto $M$ est visible de tous, gardée par le collectif qui a choisi de l'exposer sans rien faire pour en empêcher la destruction progressive (causée par des facteurs extérieurs comme le sel et la moisissure). Ce choix, dans lequel je sens un lien affectif sincère et l'exigence de protéger un trésor des influences politiques et d'une instrumentalisation, consiste, de fait, en une simple exposition qui, privée d'outils de compréhension et d'interprétation, autorise toutes les lectures simplistes, émotionnelles et trompeuses des objets eux-mêmes. La disposition de ces objets, selon des modalités improvisées ou déjà trop vues (chaussures suspendues au plafond, objets et vêtements disposés sur des étagères selon leur catégorie, comme dans les musées consacrés à l'émigration ou à I'holocauste), recrée exactement la dimension muséale fétichiste et classificatrice que nous souhaitions rejeter.

Nous pourrions discuter longtemps du changement de cap dans lequel nous nous trouvons aujourd'hui, devant les mallettes poussiéreuses et les « cris " qu'elles poussent dans une langue muette. J'écris et je raconte cette histoire aujourd'hui, non pas comme représentante d'une organisation ou d'un projet muséal en cours, mais en tant que témoin et coauteure d'une tentative de recherche partie de Lampedusa, et qui veut et peut nous donner une occasion de réflexion et de débat, autour de ce qui a constitué une aventure expérimentale et courageuse, qui s'est concrétisée par deux années de travail et qui mérite d'être racontée et analysée en tant que modèle de recherche. 